\title{
Small RNA-seq reveals novel regulatory components for apomixis in Paspalum notatum
}

\author{
Juan Pablo A. Ortiz ${ }^{1 \dagger}$, Olivier Leblanc ${ }^{2 \dagger}$, Cristian Rohr ${ }^{3}$, Mauricio Grisolia ${ }^{3}$, Lorena A. Siena ${ }^{1}$, Maricel Podio ${ }^{1}$,
} Carolina Colono ${ }^{1}$, Celeste Azzaro ${ }^{1}$ and Silvina C. Pessino ${ }^{1 *}$ (iD

\begin{abstract}
Background: Apomixis is considered an evolutionary deviation of the sexual reproductive pathway leading to the generation of clonal maternal progenies by seeds. Recent evidence from model and non-model species suggested that this trait could be modulated by epigenetic mechanisms involving small RNAs (sRNAs). Here we profiled floral sRNAs originated from apomictic and sexual Paspalum notatum genotypes in order to identify molecular pathways under epigenetic control that might be involved in the transition from sexuality to agamospermy.

Results: The mining of genes participating in sRNA-directed pathways from floral Paspalum transcriptomic resources showed these routes are functional during reproductive development, with several members differentially expressed in apomictic and sexual plants. Triplicate floral sRNA libraries derived from apomictic and a sexual genotypes were characterized by using high-throughput sequencing technology. EdgeR was apply to compare the number of sRNA reads between sexual and apomictic libraries that map over all Paspalum floral transcripts. A total of 1525 transcripts showed differential sRNA representation, including genes related to meiosis, plant hormone signaling, biomolecules transport, transcription control and cell cycle. Survey for miRNA precursors on transcriptome and genome references allowed the discovery of 124 entities, including 40 conserved and 8 novel ones. Fifty-six clusters were differentially represented in apomictic and sexual plants. All differentially expressed miRNAs were up-regulated in apomictic libraries but miR2275, which showed different family members with opposed representation. Examination of predicted miRNAs targets detected 374 potential candidates. Considering sRNA, miRNAs and target surveys together, 14 genes previously described as related with auxin metabolism, transport and signaling were detected, including AMINO ACID/AUXIN PERMEASE 15, IAA-AMIDO SYNTHETASE GH3-8, IAA30, miR160, miR167, miR164, miR319, ARF2, ARF8, ARF10, ARF12, AFB2, PROLIFERATING CELL FACTOR 6 and NITRATE TRANSPORTER 1.1.

Conclusions: This work provides a comprehensive survey of the sRNA differential representation in flowers of sexual and apomictic Paspalum notatum plants. An integration of the small RNA profiling data presented here and previous transcriptomic information suggests that sRNA-mediated regulation of auxin pathways is pivotal in promoting apomixis. These results will underlie future functional characterization of the molecular components mediating the switch from sexuality to apomixis.
\end{abstract}

Keywords: Apomixis, Apospory, Auxin, miRNA, Plant reproduction, sRNA

\footnotetext{
* Correspondence: pessino@arnet.com.ar

†Juan Pablo A. Ortiz and Olivier Leblanc contributed equally to this work.

'Instituto de Investigaciones en Ciencias Agrarias de Rosario (IICAR,

CONICET-UNR), Facultad de Ciencias Agrarias, Universidad Nacional de

Rosario, Zavalla, Argentina

Full list of author information is available at the end of the article
}

(c) The Author(s). 2019 Open Access This article is distributed under the terms of the Creative Commons Attribution 4.0 International License (http://creativecommons.org/licenses/by/4.0/), which permits unrestricted use, distribution, and reproduction in any medium, provided you give appropriate credit to the original author(s) and the source, provide a link to the Creative Commons license, and indicate if changes were made. The Creative Commons Public Domain Dedication waiver (http://creativecommons.org/publicdomain/zero/1.0/) applies to the data made available in this article, unless otherwise stated. 


\section{Background}

In plants, several small RNA pathways that likely diversified from a cellular defense mechanism against viruses and transposable elements are essential regulators of genome expression through mRNA cleavage, translational repression and DNA methylation [1]. As such, small RNAs perform critical functions in development, stress responses, and transgenerational inheritance [2]. In particular, they contribute to numerous aspects of sexual reproduction [3, 4]. Moreover, several alterations in either small RNA biogenesis, processing or targeting partially phenocopy apomixis [5], i.e. a group of peculiar reproductive behaviors leading to the formation of maternal progeny within seeds [6].

Currently, apomictic developments are viewed as the outcome of the deregulation of one or more developmental program/s involved in the sexual pathway [7], and they are broadly categorized into adventitious embryony and gametophytic apomixis [8]. Adventitious embryony relates to the spontaneous generation of somatic embryos from the maternal tissues involved in seed formation, whereas gametophytic apomixis encompasses the formation of functional, unreduced ( $2 n$ ) embryo sacs (2n-ES) from either diploid megaspore mother cells (diplospory) or nucellar companion cells (apospory) [8]. Within 2n-ES, unreduced egg cells develop parthenogenetically into maternal embryos, while the formation of viable seeds ultimately depends on endosperm development after central cell fertilization (pseudogamy) or, more sporadically, autonomous divisions [8]. Therefore, since seeds are formed in absence of meiosis and egg cell fertilization, they contain clonal embryos genetically identical to the mother plant.

Considering the prospects for the permanent fixation of any genotype regardless of genetic complexity, harnessing apomixis into agriculture would allow to dramatically accelerate plant breeding schemes and reduce the cost of improved varieties [9]. However, all major food crops reproduce sexually and attempts to introduce the trait from wild relatives in maize and pearl millet have remained unsuccessful, highlighting our lack of knowledge for apomixis molecular determinants [10-12]. Such outcome strongly contrasts with the common view of independent and repeated emergence of the trait during angiosperm evolution, therefore suggesting relatively simple controlling mechanisms [13]. A likely explanation for this is the complex nature of both apomictic plant genomes (i.e. highly heterozygous and polyploid) and apomixis-linked regions (i.e. hemizygosity, restricted recombination and heterochromatinization) ([14], and references therein). Therefore, attempts to identify candidate genes through map-based cloning approaches have proven difficult [15], however they succeeded in isolating key genes such as $B A B Y$ BOOM-like controlling parthenogenesis in aposporous Pennisetum squamulatum [16], ORIGIN RECOGNITION COMPLEX3 promoting imbalanced endosperm development in aposporous Paspalum simplex [17] or QUI GON JINN necessary for the emergence of 2n-ES in aposporous Paspalum notatum [18]. On the other hand, transcriptome comparisons between apomictic and sexual individuals have already uncovered many differentially expressed genes and several molecular pathways related to apomixis including, among others, protein degradation, transcriptional regulation, stress response, and cell-to-cell signaling [19-24].

Although a large amount of information indicates that apomixis is genetically determined [12], increasing evidence from model and apomictic species suggests that the disruption of epigenetic mechanisms participating in the control of sexual and asexual reproduction may mediate the switch between both types of reproduction [5, 25, 26]. First, 5-azacytidine induced DNA demethylation produced a significant reduction in parthenogenesis in aposporous $P$. simplex [27]. Moreover, increased sexuality was detected in diplosporous Eragrostis curvula plants subjected to environmental stress [28, 29]. Furthermore, among candidate mechanisms mediating apomixis, dosage effects or short-term epigenetic responses following polyploidization [30-32], and trans-effects generated by a non-recombinant, specific region in apomict genomes [17] have been proposed. In addition, genetic analyses in Arabidopsis thaliana and maize have shown that inactivation of members of the RNAdependent DNA Methylation (RdDM) pathway results in reproductive phenotypes reminiscent of apomictic developments [33-36]. Interestingly, in diplosporous Boechera holboelli and E. curvula RdDM members are deregulated in ovules of apomictic plants compared to sexual plants [33, 37].

These observations led several research groups to explore small RNA components in reproductive organs of sexual and apomictic plants belonging to different genera, including diplosporous Boechera [38], aposporous Hypericum and Hieracium [39, 40], and Citrus (which shows adventitious embryony) [41]. These works identified the main conserved plant miRNA families and their target genes, as well as a novel family in Citrus (miRN23-5p) [38-41]. Although deregulation of some precursors was reported, the role for miRNAs in apomixis remains elusive, since only a few targets showed a congruent expression pattern, such as the upregulation of SQUAMOSA PROMOTER BINDING PROTEIN LIKE11 and Cs9g06920 (an unknown protein containing the XS conserved domain) after miR156/157 (Boechera) and miRN23-5p (Citrus) decreased expression in apomictic ovules. Similarly, transcript expression differences between sexual and apomictic Hieracium ovules correlated poorly with 24-nt small RNAs abundances, since 
only two EXORDIUM-like genes could be identified among 40 differentially expressed targets [40].

The Paspalum genus is an attractive biological system for studying apomixis, since it is both a model system for mining candidate gene(s) and an important target forage crop [42]. The sophisticated reproductive strategy used by Paspalum spp. (i. e. a combination of sexuality, apomixis and vegetative propagation), together with the existence of a wide range of cytotypes (with ploidy levels ranging from $2 \mathrm{x}$ to $16 \mathrm{x}$ ), likely contributed to complex functional diversification and wide distribution across the Americas, from North Patagonia to Central Mexico [42]. Moreover, although Paspalum species are a key endemic forage resource for South American grasslands and pasture agro-ecosystems, they remain largely undomesticated. Therefore, generating novel knowledge on the genetic and molecular determinants of apomixis is essential for breeding and delivering novel Paspalum cultivars adapted to the challenges of climate change and societal demands on agriculture [24, 42]. To achieve this, we completed the existing transcriptomic resources available for $P$. notatum [24] with small RNA-seq profiling in florets of sexual and apomictic plants. Both transcriptomic data and genome information available in Gramineae species for miRNA gene annotation and quantitation were used to establish a comparative approach. We present evidence for differences in small RNAs profiles, including the identification of novel miRNA families, which might contribute to explain the differentiation between sexuality and apomixis.

\section{Results and discussion}

\section{Identification of Paspalum genes involved in key small} RNA pathways

Small-RNA directed pathways all rely on the activities of DICER-LIKE (DCL) enzymes that generate 21-24-nt small RNAs by processing: 1) hairpins of single-stranded RNAs synthesized from RNA POLYMERASE II (Pol II); 2) long double-stranded RNAs (dsRNAs), whose biogenesis requires transcription by Pol II or RNA POLYMERASE IV (Pol IV) followed by dsRNA synthesis by RNADEPENDENT RNA POLYMERASEs (RDRs); and 3) long dsRNAs arising from the hybridization of sense and antisense transcripts, from the fold-back of an invertedrepeat sequences, or from the hybridization of unrelated RNA molecules with sequence complementarity [1]. Following biogenesis, small RNAs are loaded onto ARGONAUTE proteins (AGO), which mediate targeting of complementary RNAs or DNAs, resulting in posttranscriptional gene silencing (PTGS; degradation through cleavage or translational repression) or RNAdependent DNA methylation (RdDM) through the recruitment of DNA methylation factors [1, 2]. Therefore, AGO proteins are central in plant small RNA pathways, owing to extensive diversification during evolution and providing specialization to the various classes of small RNAs $[1,43]$.

In order to evaluate the role of sRNA-directed pathways in apomixis, we first mined previous Paspalum reference floral transcriptome annotations [24] for genes related to sRNA biogenesis and function. Surveys for AGO rice homologs revealed 22 transcripts, which assembled into 15 genes (Additional file 1, section 1). Transcripts assembled into isotigs usually showed polymorphisms (both INDELs and SNPs) and might be allelic variants of the same gene, however some variations suggest they derive from genes existing in multiple copies in the $P$. notatum genome (i.e. isotigs of isogroup 01605). Predicted P. notatum AGO proteins (PnAGOs) were aligned with $O$. sativa Japonica and $A$. thaliana AGO proteins and a phylogenetic tree was inferred after careful visual inspection and post-processing multiple alignments for masking gaps. As shown in Fig. 1, PnAGOs distributed into the three main classes of plant AGOs. Homologs of AGO involved in meiosis (MEL1) and in reproductive cell fate specification (AGO4, AGO9) were identified. Similarly, we next identified $P$. notatum transcript homologs to most of the genes encoding proteins involved in key phases of PTGS and RdDM [44] including: Pol IV-dependent small RNA biogenesis, Pol V-dependent de novo DNA methylation, chromatin alterations and DNA methylation ( $n=67$ hits) (for details see Additional file 1, section 1). Note that, despite using less stringent criteria for filtering Blastx results, we identified no homolog for RDR2, a key member of RNA biogenesis in the RdDM pathway. Furthermore, differential expression analysis of the detected orthologs between sexual and apomictic florets in the reference transcriptome showed significant variation for four transcripts out of all identified, including: decreased expression for a transcript predicted to encode for OsAGO1D (isotig18136; $\mathrm{FDR}=0.00091$ and $\mathrm{FC}>3.24$ ) and increased expression for transcripts predicted to encode Os HISTONE DEACETYLASE 705 (isotig09390 and isotig09392; FDR $<1 \mathrm{e}-03 ; \mathrm{FC}>30$ ) and OsDCL1 (isotig27521; $\mathrm{FDR}=0.0048, \mathrm{FC}>1.8)$ in the apomictic library respect to the sexual one (Additional file 1, section $2)$. In conclusion, our analysis strongly suggests that small RNA-directed pathways are likely functional in $P$. notatum floral tissues and show differential modulation in apomictic and sexual pathways.

\section{Mapping of sRNA reads onto the $P$. notatum floral transcriptome}

Next, we decided to characterize the floral sRNA transcriptome components of an apomictic (Q4117) and a sexual (C4-4x) genotype, in mixed samples representing four developmental stages, by using a triplicate Illumina 


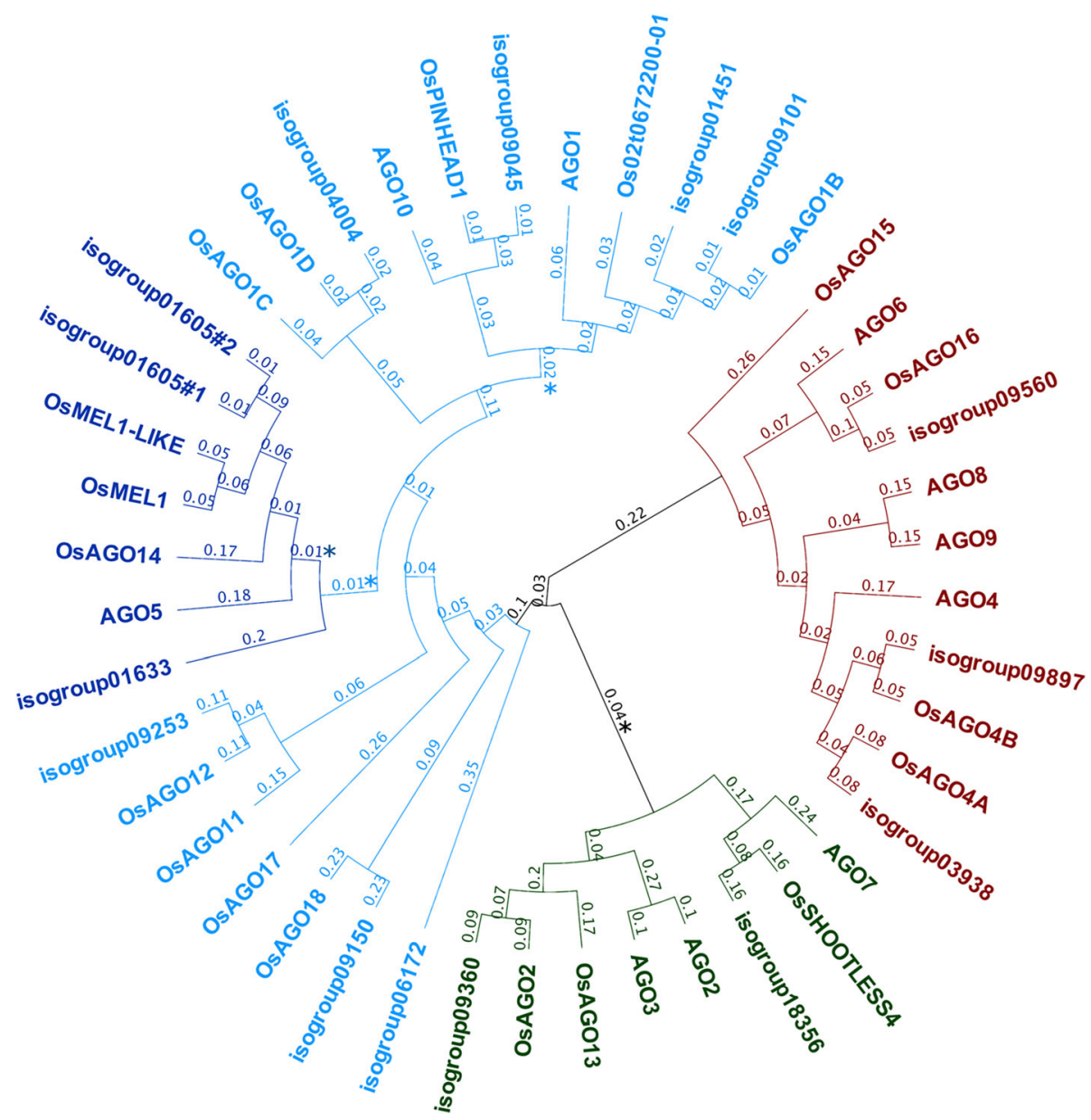

Fig. 1 Phylogenetic analysis of AGO family proteins of A. thaliana, O. sativa Japonica, and P. notatum. A. thaliana and O. sativa sequences were obtained from the Gramene database [76]. P. notatum sequences were retrieved from the corresponding floral reference transcriptome [24] Sequences alignment and neighbor-joining tree construction was performed by using Geneious 10.0.9. Branches with bootstrap support values $<50 \%$ are indicated by a star (1000 replicates). Substitution rates per site values are shown for each branch

small RNA sequencing assay. The total number of raw reads across libraries ranged from $1,558,547$ to 2,996 , 675 , with an average \% GC of $54.67 \pm 0.51$ (Additional file 2). Raw read controls revealed satisfactory quality levels, since mean Phred quality scores were between 33.2 and 38.1 along all nucleotide (nt) positions, with most sequences displaying scores between 36 and
38. A considerable number of sequences were duplicated at levels ranging from 10 to 104 and the percentage of undetermined bases at each position was near 0 for all samples. Table 1 displays the number of filtered reads (18-26 nt) for each sample. All samples showed similar length distributions, with two peaks at $21 \mathrm{nt}$ and $24 \mathrm{nt}$ (Fig. 2a).

Table 1 General statistics of the $P$. notatum sRNA reads derived from apomictic and sexual libraries

\begin{tabular}{|c|c|c|c|c|c|}
\hline Library & $\begin{array}{l}\text { Total number of } \\
\text { selected reads }\end{array}$ & $\begin{array}{l}\text { Sequences flagged as } \\
\text { poor quality }\end{array}$ & $\begin{array}{l}\text { \% Duplicated sequences } \\
\text { (average) }\end{array}$ & Sequence length & $\begin{array}{l}\text { \% GC content } \\
\text { (average) }\end{array}$ \\
\hline Apo1_S1 & $1,479,243$ & 0 & 65.4 & $18-26$ & 57.0 \\
\hline Apo2_S2 & $1,096,989$ & 0 & 60.9 & $18-26$ & 56.0 \\
\hline Apo3_S3 & 810,471 & 0 & 59.2 & $18-26$ & 56.0 \\
\hline Sex1_S4 & $1,162,189$ & 0 & 64.5 & $18-26$ & 58.0 \\
\hline Sex2_S5 & $1,007,577$ & 0 & 61.7 & $18-26$ & 57.0 \\
\hline Sex3_S6 & $1,169,125$ & 0 & 62.9 & $18-26$ & 57.0 \\
\hline
\end{tabular}




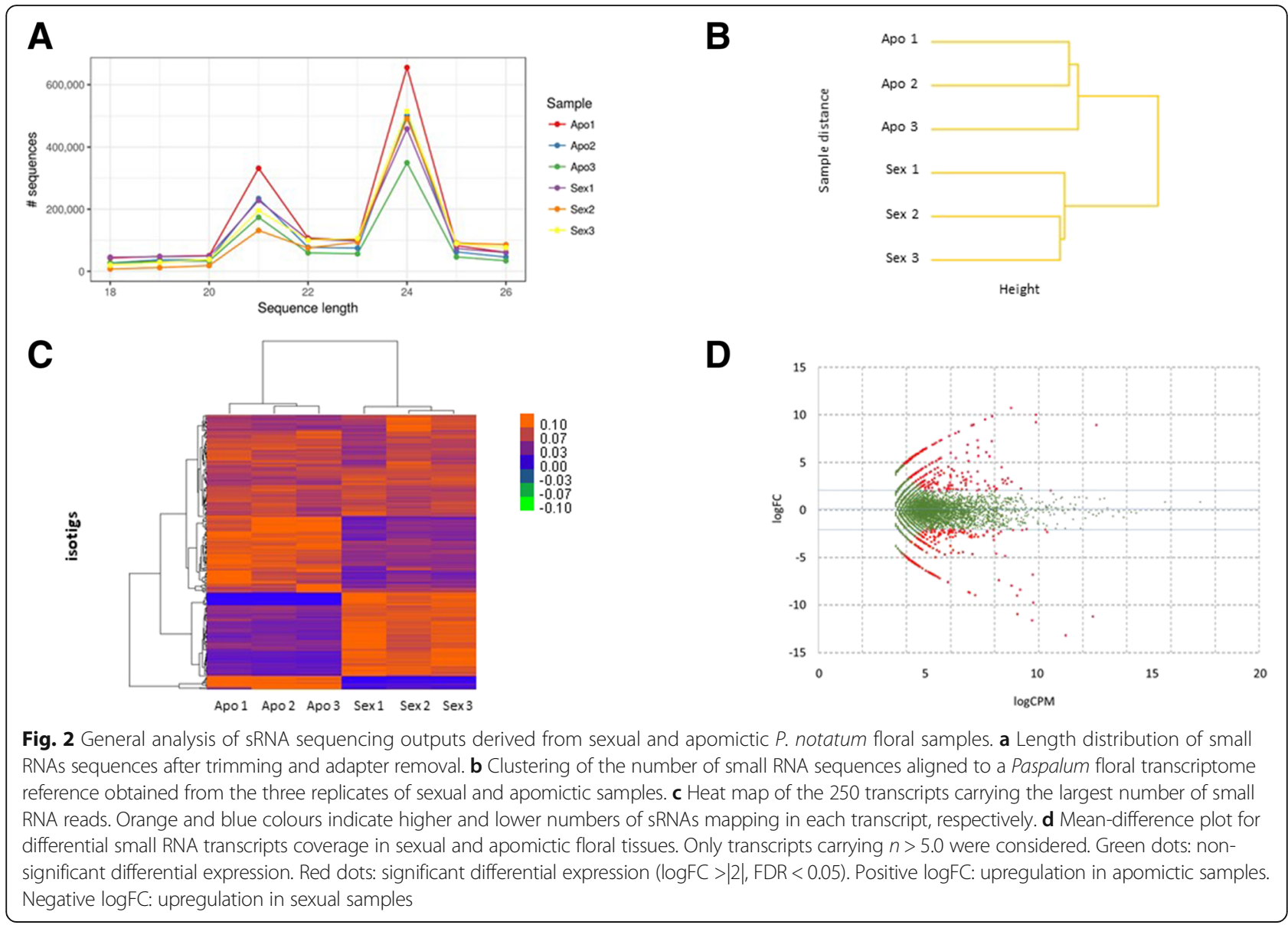

As no reference genome is available for $P$. notatum, small RNA reads were mapped onto a Paspalum reference transcriptome generated using RNA samples extracted from developing spikelets (premeiosis, meiosis, postmeiosis, anthesis) collected from the two genotypes used in this work (Q4117 and C4-4x) [24]. In particular, this reference was assembled using 454/Roche FLX+ sequences. It contains 48,842 isogroups (genes), out of which $18 \%$ had at least two isotigs (alleles) and was validated for $\sim 100$ sequences previously characterized. Based on these results, this reference likely represents a comprehensive resource that reflects transcripts variation and occurrence. Therefore, we assume that its use greatly helped to contend the constraints resulting from the polyploid and highly heterozygous nature of Q4117 and C4-4x during mapping and subsequent sRNA representation comparisons. Moreover, since both genotypes used in the analysis are tetraploid, the polyploidy status should not interfere with results. Only $21-23 \%$ of the reads could be mapped using the Bowtie aligner, indicating that a large fraction of the sRNAs derived from sequences with low expression in flowers or corresponded to non-silencing small RNAs (Table 2). The average numbers of mapped small RNA reads on apomictic and sexual libraries were equivalent $(216,543.66 \pm 53,362.90$ and 207,796.00 $\pm 18,421.28$, respectively; $t=0.831$, paired samples) and clustering analysis revealed high similarities between replicates (Fig. 2 b). Out of 67,617 reference transcripts (assembled into 48,769 genes), 12,942 (19.14\%) were targeted by small RNA sequences $(n \geq 5.0$ considering all libraries) and thus selected for further analyses. The number of reads mapped in this set of transcripts for each library is provided (Additional file 3, section 1) together with the list of the top 1000 transcripts showing the highest read coverage (Additional file 3, section 2). Within the most represented targets, clustering revealed differences in the number of mapped

Table 2 Mapping of sRNA reads from apomictic and sexual libraries onto the Paspalum transcriptome reference [24]

\begin{tabular}{lll}
\hline Sample name & \% aligned & Millions of reads aligned \\
\hline Apo1_S1 & 22.6 & 0.3 \\
Apo2_S2 & 22.4 & 0.2 \\
Apo3_S3 & 22.6 & 0.2 \\
Sex1_S4 & 22.9 & 0.3 \\
Sex2_S5 & 21.0 & 0.2 \\
Sex3_S6 & 21.7 & 0.3 \\
\hline
\end{tabular}


reads occurring in both directions $(i . e$. a significant higher number of sRNA reads in the apomictic or the sexual libraries) (Fig. 2 c).

Many genes involved in reproduction have been identified in the Paspalum reference transcriptome. Next, we focused in determining to which extent small-RNA target genes belong to this particular group. To achieve this, we first categorized $O$. sativa orthologs of annotated small RNA target genes (4560 unique genes) using the plant structure development stage ontologies provided Plant Ontology database [45]. This revealed numerous genes with ontologies associated with male and female reproduction (e.g. androecium development stage, gametophyte development stage, inflorescence development stage, mature plant embryo stage, plant embryo development stage, pollen mother cell meiosis, reproductive shoot system development stage, reproductive shoot system development stage, and sporophyte reproductive stage) (Additional file 3, section 3). Considering GO ontologies, only a few families were highly represented in the Paspalum reference transcriptome: within the class 'biological processes', the subclasses 'cellular process' (GO: 0009987) and 'metabolic process' (GO: 0008152) resulted predominant, representing 29.3 and $28.8 \%$ of the transcripts, respectively; within the class 'cellular component', the most represented subclasses were 'cell part' (GO: 0044464) and 'organelle/nucleus' (GO: 0043226), with 39.6 and $29.1 \%$ of the transcripts, respectively. Moreover, within the class molecular function, the subclasses 'catabolic activity' (GO: 0003824) and 'binding' (GO: 0005488) reached 50 and $27.8 \%$ of the total transcripts, respectively (Additional file 3, section 4, panel A). Analysis for overrepresentation in target genes compared to the full floral transcriptome showed significant enrichment for these families or related subfamilies (Additional file 3, section 4, panel B).

Next, edgeR was used to compare the number of sRNA reads between sexual and apomictic libraries that map over all Paspalum floral transcripts. Counts and edgeR scores are detailed in Additional file 3, section 5, and pictured in Fig. 2 d. Using an FDR cut-off value of 0.05 , we identified 1525 transcripts showing significant differences. These transcripts split evenly into two groups, including those that match sRNAs overrepresented in apomictic samples (753) or in sexual samples (772). However, if we consider only those transcripts displaying larger differences ( $>$ four-fold change), a higher number match sRNAs overrepresented in apomictic samples compared to sexual ones (480 vs $384 ; p=$ 0.0014, Pearson's chi-squared test). Interestingly, among all differential transcripts, we detected several homologs of $O$. sativa genes encoding proteins involved in: 1) meiosis: MEIOSIS-SPECIFIC DNA RECOMBINASE, SYNAPTONEMAL COMPLEX (SC) ASSEMBLY; MALE-
GAMETE-SPECIFIC HISTONE H3; 2) biomolecules transport: AMINO ACID/AUXIN PERMEASE 15; MULTIDRUG RESISTANCE 9; MRS2/MGT FAMILY MEMBER 9; ABC TRANSPORTER I FAMILY MEMBER 8; MONOSACCHARIDE TRANSPORTER 1; SORBITOL TRANSPORTER; AMMONIUM TRANSPORTER 3 MEMBER 1; METAL TOLERANCE PROTEIN $\quad 8.1 ; \quad$ HIGH-AFFINITY POTASSIUM(K+) TRANSPORTER 13; AMINO ACID TRANSPORTERLIKE 5; and BI-DIRECTIONAL AMINO ACID TRANSPORTER 1 ; 3) hormone signaling pathways: AMINO ACID/AUXIN PERMEASE 15; MITOGENACTIVATED PROTEIN KINASE KINASE KINASE; GIBBERELLIN-INSENSITIVE DWARF 2; INDOLE-3ACETIC ACID (IAA)-AMIDO SYNTHETASE GH3-8; and IAA30; 4) transcriptional regulation: b-ZIP TRANSCRIPTION FACTOR 71; ETHYLENE RESPONSE FACTOR 77 (APETALA-2-LIKE TRANSCRIPTION FACTOR); FINGER PROTEIN WZF1; WRKY GENE 67; BEL1-LIKE HOMEODOMAIN GENE 6; MADS27; and GROWTH-REGULATING FACTOR 1, 3, 9, and 10; and 5) cell cycle and proliferation: CELL DIVISION CYCLE PROTEIN 20.1; and CELL DIVISION CONTROL PROTEIN 2 HOMOLOG 2 (Additional file 3, section 5). In summary, mapping small RNA reads onto the Paspalum reference transcriptome revealed that numerous transcripts homologous to $O$. sativa genes associated with plant reproductive development, plant hormone (auxin/ cytokinin) signaling, biomolecules transport, transcription control and cell cycle undergo differential epigenetic regulation during apomictic and sexual reproductive development.

Previous comparative transcriptome analyses of apomictic and sexual $P$. notatum floral reference libraries (454/ Roche FLX +) revealed a list of 3732 (out of 67,617 ) isotigs associated with apomixis [24], which indicated that global differential expression comprises $5.51 \%$ of the total transcripts. Here, we found 1525 transcripts displaying differential sRNA representation, which accounts for $2.25 \%$ of the total 67,617 reference library isotigs. According to these data, apparently $40.8 \%$ of differential expression could be assigned to the occurrence of silencing events. However, a correlation between the two comparisons (differential sRNA and long transcript representation in apomictic vs. sexual samples) is not strictly linear, because they were originated from different experimental designs. There is a contrasting output capacity inherent to both techniques: while Illumina produces massive quantity of short reads, 454/Roche FLX + generates longer reads, but many of them are represented by a low number. High output facilitates the discovery of statistically significant differential representation, because of the higher sample size (n) used in comparisons, which might lead to a consequent overestimation of the percentage of differentially expressed genes being 
targeted by silencing mechanisms. An accurate estimation of the percentage of differentially expressed genes subjected to silencing would require the mapping of sRNA reads onto floral Illumina reference libraries, which are currently not available for $P$. notatum. Besides, since our libraries originated from bulks, our approach did not allow to discriminate at which specific stage differential expression occurred. Nevertheless, we likely detected differential expression across all developmental stages and further investigations such as in situ hybridization or qPCR experiments should help in resolving temporal differences.

\section{Discovery of Paspalum miRNA genes}

To search for miRNAs expressed during reproductive development in $P$. notatum, we computed small RNA sequences with ShortStack using the Paspalum floral transcriptome and the closely related genomic sequences of O. sativa, Setaria italica and Sorghum bicolor as references. Percentages of unmapped reads varied from 58.0\% in S. bicolor to $69.7 \%$ in P. notatum (Table 3). Among mapped reads, the highest proportion of uniquely mapped sequences was detected for the $P$. notatum transcriptome (16.7\%), while it dropped down to $8.0 \%$ for heterologous genomic references (Table 3). This result was anticipated, since the Paspalum transcriptome reference displays less sequence variability and redundancy than heterologous genomic references. We detected a maximum of small RNA clusters in S. italic and a minimum in rice (Table 3), most of them producing DicerCall scores (i.e. mature small-RNAs lengths) inconsistent with Dicer endoribonuclease activity (Fig. 3). Interestingly, ShortStack analysis revealed no evidence for the occurrence of phased small RNAs (PhaseScore $<10$ for 21 and 24-nt reads) across the four references used.

Computational analysis for hairpin configuration detected 8, 35, 42 and 42 putative miRNA precursors over $P$. notatum, O. sativa, S. italica and S. bicolor references, respectively (Table 3 ). Candidate miRNA genes mainly generated mature miRNA sequences of 21-22 nt (Fig. 3) . The corresponding transcripts of $P$. notatum and the genomic locations in reference genomes of all precursors, as well as, the length and the sequence of the major mature miRNAs detected are shown in Additional file 4,

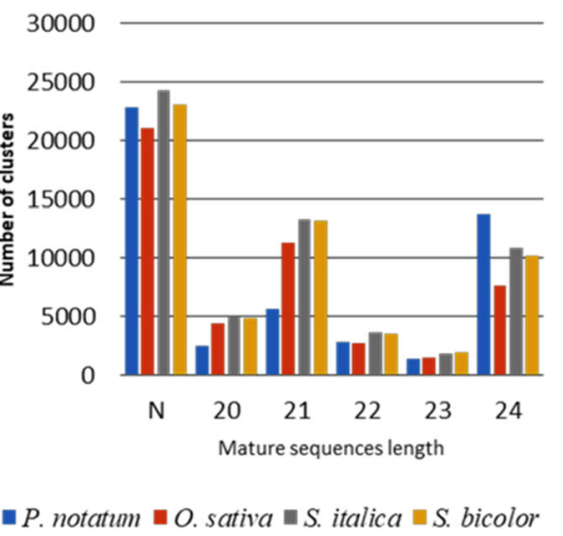

Fig. 3 Histogram of length distribution of sRNA sequences. Sequences were associated to predicted clusters using four references. $\mathrm{N}$ : lengths inconsistent with DICER activities

section 1. Precursor coverage ranged from 3 to 221,108 short reads, and from 2 to 213,532 for major mature sequences. Three candidate clusters were eliminated for further analyses due to low read coverage $(n \leq 5)$. The resulting 124 miRNA precursors distributed over all chromosomes of the three genomic references but rice chromosome 11, S. bicolor chromosome 5, and S. italica chromosome 8 (Additional file 4, section 1). Interestingly, two putative miRNA precursors detected using the rice genomic reference (Cluster_9305 and Cluster_9306) mapped within a region of chromosome 12 previously identified as syntenic to the region associated with apomixis in several Paspalum species [42, 46, 47].

\section{Identification of conserved and novel miRNAs expressed in the floral transcriptome of $P$. notatum}

To identify conserved and novel miRNAs expressed in the floral transcriptomes of sexual and apomictic $P$. notatum, mature miRNA sequences were searched in MirBase [48] using the BLAST SSEARCH option and an E-value $<0.05$. The 124 predicted sequences generated 40 unique conserved miRNA accessions, corresponding to 22 miRNA families, including miR156, miR166, miR164, miR167, miR171, miR396, and miR2275 as the most abundant ones (Table 4; Additional file 4, section

Table 3 Summary of primary floral P. notatum sRNA alignment over four references using ShortStack v.5

\begin{tabular}{|c|c|c|c|c|}
\hline \multirow[t]{2}{*}{ Reads alignments } & \multicolumn{4}{|l|}{ References } \\
\hline & $\begin{array}{l}\text { P. notatum } \\
\text { transcriptome }\end{array}$ & $\begin{array}{l}\text { O. sativa } \\
\text { genome }\end{array}$ & $\begin{array}{l}\text { S. bicolor } \\
\text { genome }\end{array}$ & $\begin{array}{l}\text { S. italica } \\
\text { genome }\end{array}$ \\
\hline Uniquely mapped (\%) & 16.4 & 6.5 & 8.0 & 7.4 \\
\hline Multi-mapped (\%) & 13.9 & 23.9 & 34.0 & 31.9 \\
\hline Unmapped (\%) & 69.7 & 69.6 & 58.0 & 60.7 \\
\hline Numbers of clusters & 49,1 & 48,8 & 56,9 & 58,8 \\
\hline Number of miRNA precursors & 8 & 35 & 42 & 42 \\
\hline
\end{tabular}


2). Most families had accessions detected in three out of the four references $(n=17)$, but three of them (miR167, miR396 and miR2275) were found across all four references (Table 4).

In addition, eight putative new mature miRNA sequences (i.e. with no significant match in MirBase) were detected (Table 4). Folding analysis of their putative precursors (named Pn_miR1 to Pn_miR8) revealed that all hairpin structures with the Dicer cleavage sites within the arm of the stem regions displayed the expected match between miRNAs and opposite miRNAs" and exhibited minimum free energy $<60 \mathrm{kcal} . \mathrm{Mol}^{-1}$ (Additional file 5). Thus, these 8 miRNA mature sequences were considered as novel plant miRNAs. Precursors of Pn_miR1, Pn_miR3, Pn_miR5, Pn_miR6, Pn_miR7 and $\mathrm{Pn} \_$miR8 were found using genome references only. $\mathrm{Pn}$ miR4 was detected using the $P$. notatum transcriptome and the S. bicolor and S. italic genomes. In contrast, Pn miR2 was found using the $P$. notatum transcriptome only, which suggests that it might be specific to $P$. notatum. Finally, a phylogenetic analysis considering all unique mature miRNA sequences detected in our analysis showed that Pn_miR3, Pn_miR4 and Pn_miR5 are evolutionarily related to miR156, while Pn_miR7 is related to miR171 (Additional file 6). Association of the remaining four Pn_miRNAs with conserved accessions could not be supported by the bootstrapping analysis $(<$ $65 \%)$, suggesting they might be novel plant miRNAs.

\section{Differentially expressed miRNA genes}

Small RNA read counts derived from both apomictic and sexual libraries were normalized and analyzed for differential expression using edgeR. Out of the 124 candidate miRNA precursors, 9 showed highly significant differences between reproductive modes, being all of them overrepresented in apomictic libraries $(\operatorname{logFC} \geq 2.0$; FDR $<$ 0.05). According to our annotation, these loci produce both conserved (miR167/MIMAT0000196: Cluster_5800, _9305 and _20699; miR171/MIMAT0037190: Cluster_ 29775; miR2275/MIMAT0037125: Cluster_43159 and _34865) and novel (Pn_miR1: Cluster_3423; Pn-miR2: Cluster_13846; Pn-miR8: Cluster_13322) miRNAs (Additional file 4, section 3). In addition, we identified 56 clusters with moderate but significant differences in coverage $(1<\log \mathrm{FC}<2, \mathrm{FDR}<0.05)$ corresponding to various families, including: miR156, miR159, miR160, miR164, miR167, miR169, miR171, miR319, miR390, miR393, miR396, miR529, and Pn_miR6. Again, most precursors showed increased expression in apomict libraries except for three clusters all containing a miR2275 accession (Additional file 4, section 3; Fig. 4).

Several of the miRNAs showing strong and modest differences in abundance between apomictic and sexual plants have been associated with many aspects of plant reproductive development, including: miR156, miR159, miR160, miR167, miR169, miR319, miR390, miR2118, and miR2275 [49-51]. Of interest, in our analysis, athmiR167a-5p precursors detected using the three genome references were overexpressed in apomictic samples while precursors for a second member of the miR167 family (ccl-miR167a) showed a modest increase using the $P$. notatum reference transcriptome and two of the genomic references (Additional file 4, section 3; Fig. 4). Thus, miR167 precursors, two of which mapped on a rice segment of chromosome 12 syntenic to the apomixis controlling locus in $P$. notatum, appeared consistently upregulated in apomictic libraries compared to sexual ones. Alike miR167 precursors, most of the remaining families (for instance miR156, miR160, miR166, miR171, miR319, miR396, miR529), exhibited congruent expression patterns among the various precursors detected across the references used. However, the four precursors we identified for the miR2275 family showed contrasting behavior: while no significant change was detected for osa-miR2275a and bdi-miR2275a (specifically detected in P. notatum and S. italica, respectively), the two remaining precursors showed opposite changes, i.e. increased expression in apomictic libraries for ata-miR2275b-3p (for both O. sativa and S. italica references) and slightly decreased expression in apomictic libraries for zma-miR2275b-5p (for both S. italica and S. bicolor references).

\section{Prediction of miRNA target genes and putative miRNA- dependent pathways for installing apomixis}

With the aim of identifying putative miRNAs targets, we computationally predicted binding sites for the 40 mature miRNAs reported in Table 4 in the Paspalum transcriptome reference using TargetFinder. Using a cut off value of 4.0, 376 hits were identified representing 283 different transcripts, out of which 224 were unique hits (i.e. target of a single miRNA), while $40,11,5$, and 3 were found 2, 3, 4 and 5 times, respectively (Additional file 7 , section 1 ). Most multiple-hits transcripts $(n=48)$ were targeted by miRNAs from a single family, but a few ones were targeted by members of two families (miRNA156/miR529; miR159/miR319; miR160/miR166; miR396/Pn_miR1, and; Pn_miR1/Pn_miR2). We identified targets for all predicted miRNA families but miR158. The number of targets varied greatly across families (from 1 to 78 for miR156) and accessions (from 1 to 19 for Pn_miR2) (Fig. 5 a). BLASTX against rice reference peptides produced an annotation for 275 (73.3\%) of the 374 transcripts, representing 195 unique genes (Additional file 7, section 1). Target annotations were consistent with previous reports for target-miRNA regulatory modules, as illustrated by the occurrence of homologies to transcription factors of the SQUAMOSA 
Table 4 Unique miRNA mature sequences present on the floral transcriptome of sexual and apomictic $P$. notatum

\begin{tabular}{|c|c|c|c|c|c|c|c|}
\hline miRNA mature sequence & $\mathrm{nt}$ & Accession* & $\mathrm{Pn}$ & Os & $\mathrm{Sb}$ & $\mathrm{Si}$ & Family \\
\hline GCUCACUUCUCUUCCUGUCAGC & 22 & MIMAT0015124 & 0 & 0 & 0 & 1 & miR156 \\
\hline GCUCACUUCUCUCUCUGUCAGU & 22 & MIMAT0015129 & 0 & 0 & 1 & 0 & miR156 \\
\hline GCUCACUGCUCUGUCUGUCAUC & 22 & MIMAT0015128 & 0 & 1 & 1 & 1 & miR156 \\
\hline UUGACAGAAGAGAGUGAGCAC & 21 & MIMAT0026186 & 0 & 1 & 0 & 0 & miR156 \\
\hline UUUGGAUUGAAGGGAGCUCUG & 21 & MIMAT0001023 & 0 & 1 & 1 & 1 & miR159 \\
\hline UGCCUGGCUCCCUGUAUGCCA & 21 & MIMAT0000178 & 1 & 0 & 2 & 2 & miR160 \\
\hline UGGAGAAGCAGGGCACGUGCA & 21 & MIMAT0000185 & 0 & 1 & 1 & 1 & miR164 \\
\hline UGGAGAAGCAGGGCACGUGCU & 21 & MIMAT0001034 & 0 & 1 & 1 & 1 & miR164 \\
\hline CAUGUGCCCAUCUUCUCCACC & 21 & MIMAT0015138 & 0 & 1 & 0 & 0 & $\operatorname{miR} 164$ \\
\hline CACGUGCUCCCCUUCUCCACC & 21 & MIMAT0015319 & 0 & 1 & 0 & 0 & miR164 \\
\hline UCGGACCAGGCUUCAUUCCUC & 21 & MATAT0001072 & 0 & 1 & 1 & 1 & $\operatorname{miR} 166$ \\
\hline UCGGACCAGGCUUCAUUCCCC & 21 & MIMAT0000189 & 0 & 5 & 5 & 5 & miR166 \\
\hline UCGGACCAGGCUUCAAUCCCU & 21 & MIMAT0001037 & 0 & 1 & 2 & 2 & miR166 \\
\hline UGAAGCUGCCAGCAUGAUCUGA & 22 & MIMAT0014072 & 1 & 1 & 1 & 0 & miR167 \\
\hline UGAAGCUGCCAGCAUGAUCUA & 21 & MIMAT0000196 & 0 & 1 & 1 & 2 & miR167 \\
\hline UCGCUUGGUGCAGAUCGGGACC & 22 & MIMAT0001045 & 0 & 1 & 1 & 1 & miR168 \\
\hline UGGGCGGUCACCUUGGCUAGC & 21 & MIMAT0026431 & 0 & 0 & 1 & 1 & miR169 \\
\hline UUGAGCCGCGUCAAUAUCUCC & 21 & MIMAT0014082 & 0 & 1 & 1 & 1 & miR171 \\
\hline GGAUAUUGGUGCGGUUCAAUC & 21 & MIMAT0022877 & 0 & 1 & 0 & 0 & miR171 \\
\hline GUGGUAUUGUUCCGGCUCAUG & 21 & MIMAT0037190 & 0 & 1 & 1 & 1 & miR171 \\
\hline UUGGACUGAAGGGUGCUCCCU & 21 & MIMAT0020761 & 0 & 1 & 1 & 1 & miR319 \\
\hline AAGCUCAGGAGGGAUAGCGCC & 21 & MIMAT0000931 & 0 & 1 & 0 & 0 & miR390 \\
\hline UCCAAAGGGAUCGCAUUGAUC & 21 & MIMAT0000957 & 1 & 1 & 1 & 0 & miR393 \\
\hline GUUCUCCUCAAGCACUUCACA & 21 & MIMAT0015347 & 0 & 1 & 0 & 1 & miR395 \\
\hline UCCACAGGCUUUCUUGAACUG & 21 & MIMAT0001601 & 0 & 0 & 0 & 1 & miR396 \\
\hline UUCCACAGCUUUCUUGAACUG & 21 & MIMAT0000944 & 2 & 1 & 1 & 1 & miR396 \\
\hline UUCCACAGCUUUCUUGAACUU & 21 & MIMAT0000945 & 0 & 1 & 0 & 1 & miR396 \\
\hline UUGAGUGCAGCGUUGAUGAGC & 21 & MIMAT0025996 & 0 & 0 & 1 & 1 & miR397 \\
\hline UGUGUUCUCAGGUCGCCCCCG & 21 & MIMAT0014020 & 0 & 0 & 1 & 0 & miR398 \\
\hline UGCCAAAGGAGAGUUGCCCUG & 21 & MIMAT0000952 & 0 & 0 & 1 & 1 & miR399 \\
\hline UGCCAAAGGAGAGCUGCCCUG & 21 & MIMAT0000992 & 0 & 1 & 1 & 1 & miR399 \\
\hline UGGAAGGGGCAUGCAGAGGAG & 21 & MIMAT0014028 & 0 & 1 & 1 & 1 & miR528 \\
\hline AGAAGAGAGAGAGUACAGCCU & 21 & MIMAT0014030 & 0 & 1 & 1 & 1 & miR529 \\
\hline UGGGUGUCAUCUCUCCUGGAGC & 22 & MIMAT0015366 & 0 & 0 & 1 & 0 & miR1432 \\
\hline UUCCCGAUGCCUCCCAUUCCUA & 22 & MIMAT0011741 & 0 & 0 & 1 & 0 & miR2118 \\
\hline UUCCUGAUGCCUCCCAUUCCUA & 22 & MIMAT0011745 & 0 & 0 & 0 & 1 & $\operatorname{miR} 2118$ \\
\hline UUUGGUUUCCUCCAAUGUCUCA & 22 & MIMAT0035533 & 0 & 0 & 0 & 1 & miR2275 \\
\hline CUUGUUUUCCUCCAAUGUCUCA & 22 & MIMAT0037125 & 0 & 1 & 0 & 1 & $\operatorname{miR} 2275$ \\
\hline CUUGGUUCCCUCCAAUAUCUCA & 22 & MIMAT0011758 & 1 & 0 & 0 & 0 & miR2275 \\
\hline AGGAUUAGAGGGAACUGAACC & 21 & MIMAT0011769 & 0 & 1 & 1 & 1 & $\operatorname{miR} 2275$ \\
\hline CUCUCGCCGGCGUGCGCACUCC & 22 & no hit & 0 & 0 & 1 & 0 & Pn_miR1 \\
\hline AGUGCGCCGCCGUCGAUCUGC & 21 & no hit & 1 & 0 & 0 & 0 & Pn_miR2 \\
\hline UUGACAGAAGAGAGCGAGCAC & 21 & no hit & 0 & 0 & 1 & 1 & Pn_miR3 \\
\hline UGACAGAAGAGAGUGAGCAC & 20 & no hit & 1 & 0 & 2 & 1 & Pn_miR4 \\
\hline
\end{tabular}


Table 4 Unique miRNA mature sequences present on the floral transcriptome of sexual and apomictic P. notatum (Continued)

\begin{tabular}{|c|c|c|c|c|c|c|c|}
\hline miRNA mature sequence & $\mathrm{nt}$ & Accession* & $\mathrm{Pn}$ & Os & $\mathrm{Sb}$ & $\mathrm{Si}$ & Family \\
\hline UGACAGAAGAGAGAGAGCAC & 20 & no hit & 0 & 0 & 1 & 1 & Pn_miR5 \\
\hline GGGCAAAUCAUCUGGGCUACC & 21 & no hit & 0 & 0 & 0 & 1 & Pn_miR6 \\
\hline UGAGCCGAGCCAAUAUCACUUC & 22 & no hit & 0 & 1 & 1 & 1 & Pn_miR7 \\
\hline UAUUGUCUCGGCUCACUCAGA & 21 & no hit & 0 & 1 & 2 & 2 & Pn_miR8 \\
\hline
\end{tabular}

Sequences were detected by using four references: Pn: Paspalum transcriptome; Os: Oryza sativa Japonica genome; Sb: Sorghum bicolor genome; Si: Setaria italica genome

*Accession number was obtained by performing BLASTn analysis (SSEARCH option) of mature sequences on miRBase [48]

PROMOTER BINDING PROTEIN LIKE (SPL) family (miR156), the NAC (NAM, ATAF1/2, and CUC2) family (miR164), the HD-ZIP family (miR166), the GROWTHREGULATING FACTOR family (miR396), and the AUXIN RESPONSE FACTOR (ARF) family (miR160, miR167) [52]. The most represented GO annotations for the 196 O. sativa orthologs corresponding to molecular function (MF), cellular components $(\mathrm{CC})$, and biological processes (BP) are shown in Fig. 5 b, c and d, respectively. For MF ontologies (60 hits), genes categorized into catalytic activity (43.2\%) and binding (32.1\%) (Fig. 5 b). Within the CC group (73 hits; Fig. 5b), genes associated with cell parts $(39.9 \%)$ and organelles $(32.7 \%)$ were the most abundant (Fig. 5 c). Likewise, within the BP class (103 hits) cellular processes and metabolic processes (37.5 and $32.4 \%$, respectively) were also highly represented (Fig. $5 \mathrm{~d}$ ).

Next, to identify putative major molecular routes differentially modulated by miRNAs during sexual and apomictic developments, we mined the Paspalum

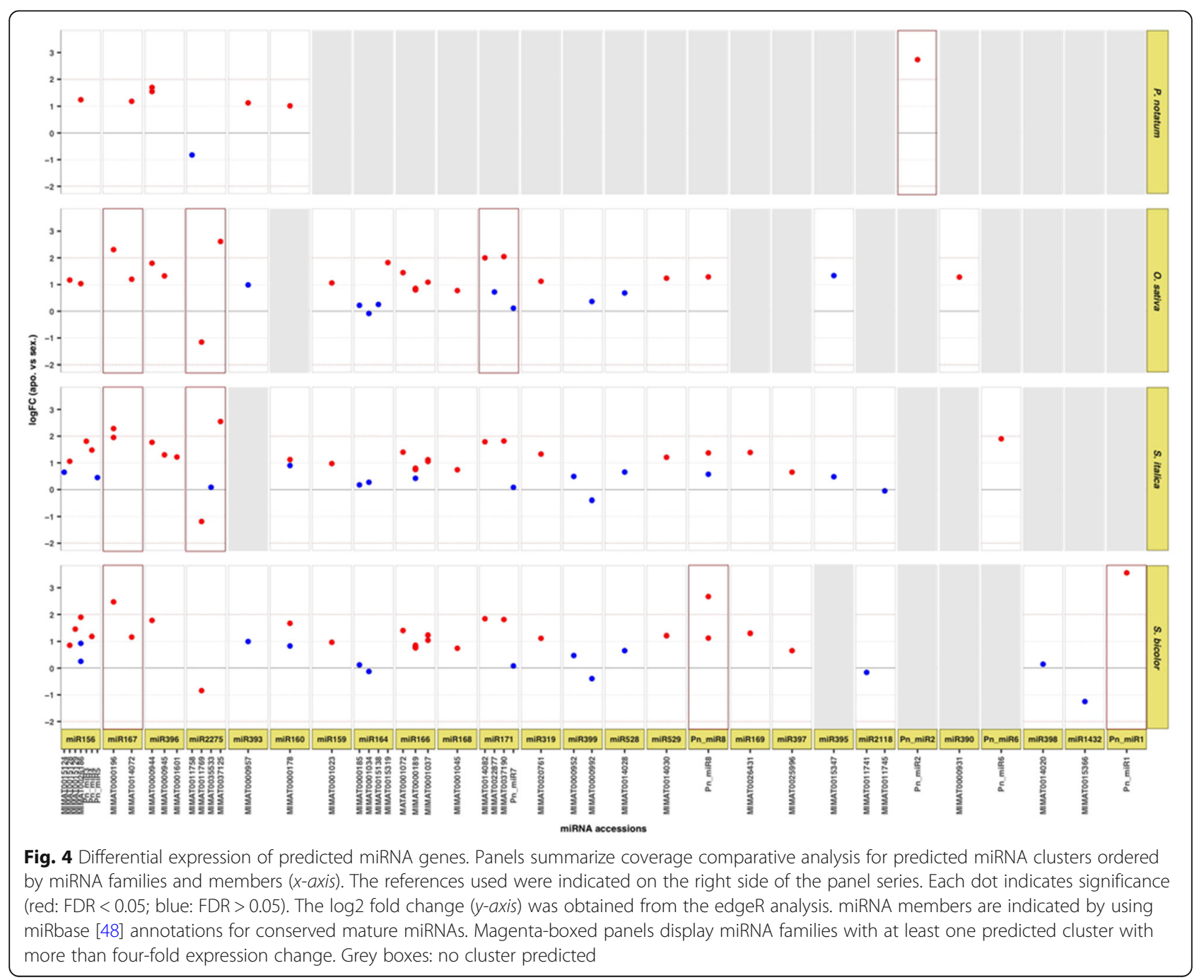




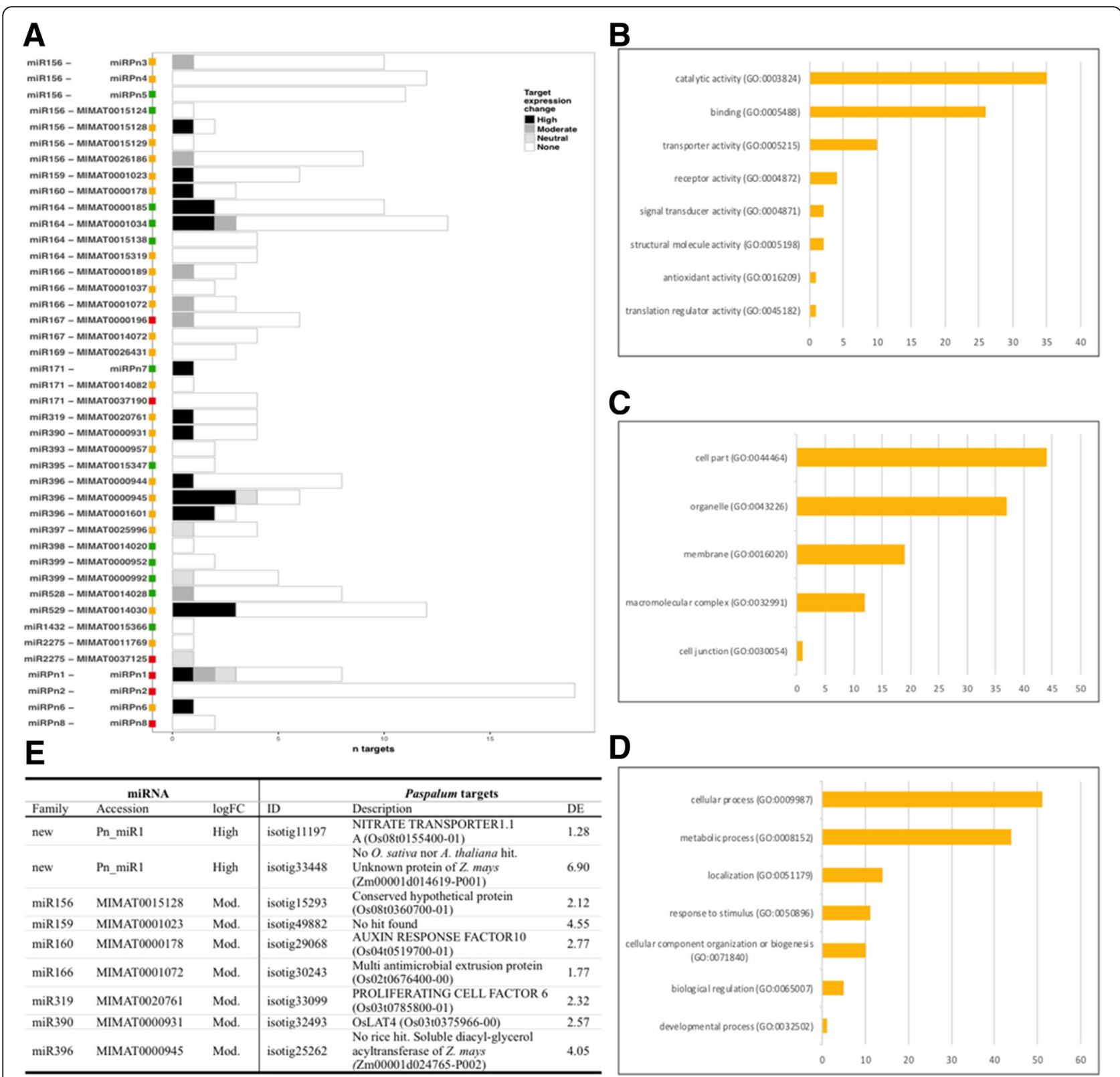

Fig. 5 Candidate miRNA-regulated genes expressed during $P$. notatum reproductive development. a. Number of target genes by miRNA families. Colours in bars indicate the number of targets showing high, moderate, neutral and no change (see insert box for legend). Square boxes at the left indicate the differential expression of miRNA precursors between apomictic and sexual sRNA libraries: green: no change; orange: moderate change $(1<\log F C<2)$; red: high change (logFC $>2$ ). b-d Significantly enriched GO classes corresponding to Molecular Function (b), Cell Component (c) and Biological Process (d). $\mathbf{f}$ Candidate target-miRNA regulatory modules for licensing apomixis

reference transcriptome for candidate target transcripts already assessed for differential expression between apomixis and sexuality [24]. Out of 374 predicted target transcripts, 207 could be analyzed ( $\mathrm{n}$ reads $>10$ in at least one sample) and a significant difference $($ FDR $<0.05)$ was found for 34 transcripts (17.6\%) targeted by members of 14 miRNA families. As shown in Fig. 5 a, high fold changes prevailed $(n=21 ; \mathrm{FC}>4)$ over moderate $(n=6$; $1<\mathrm{FC}<2)$ and low $(n=5 ; \mathrm{FC}<1)$ differences. However, despite increased expression in apomictic libraries affected most miRNA genes differentially expressed between apomictic and sexual libraries, no bias in direction change was observed for the predicted targets (18 downregulated vs. 16 upregulated).

Then, miRNA/target pairs with consistent opposed expression were identified by comparing changes in representation for miRNAs and their target transcripts (Additional file 7, section 2). Considering $\log \mathrm{FC}>2$, we 
detected one miRNA displaying an increased expression congruent with a transcriptional decrease of its targets: Pn_miR1 (targets: isotig11197and isotig33448, encoding NITRATE TRANSPORTER1.1A and the unknown protein Zm00001d014619-T001, respectively) (Fig. 5 e). Nevertheless, as the regulatory impact of miRNAs does not only depend on their expression levels, but also on their target mRNA configuration [53], we also considered targets of miRNAs whose precursors showed moderate changes $(1<\operatorname{logFC}=<2, \mathrm{FDR}<0.05)$. By doing that, we found seven additional genes with complementary expression patterns, showing increased expression of miRNA precursors and decreased expression of the targets. Five of them encode for homologs to plant proteins with assigned functions including: the ARF10 and PROLIFERATING CELL FACTOR 6 (TEOSINTE BRANCHED1/ CYCLOIDA/PROLIFERATING CELL NUCLEAR ANTIGEN FACTOR1 or TCP family) transcription factors, and three proteins related to cellular activity (OsLAT4, Zm00001d024765-P002, and Os02t0676400-00) (Fig. 5 e). The function of the two latter ones remains unknown and one of them produced no BLASTX hit against nonredundant protein sequences (Fig. 5 e).

ARFs (auxin response factors) are key proteins involved in many developmental processes through the regulation of auxin responsive gene expression by targeting specific binding sites within their promoter $[54,55]$. The current working model for auxin perception and signaling stipulates ARF inhibition at low auxin levels via dimerization with AUX/IAA and TOPLESS recruitment, whereas higher levels of auxins allows ARF activation by ubiquitinization of AUX/IAAs, which bind to the $\mathrm{SCF}^{\mathrm{TIR} / \mathrm{AFB}}$ complex [56]. Recent evidence shows that several of the numerous $A R F$ genes present in the plant genomes are post-transcriptionally regulated by small RNAs; interestingly, a critical role of auxin in controlling female gametophyte differentiation was reported in Arabidopsis [57] and miR160 was reported as downregulated before fertilization in rice ovules while three of its targets (OsARF10, OsARF18 and OsARF22) increase in expression during female gametophyte formation [58]. Furthermore, TCP transcription factors (including PROLIFERATING CELL FACTOR 6) are central in establishing a link between cell proliferation and hormone response, and they are also involved in many aspects of plant development, including reproduction $[59,60]$. TCP6 belongs to Class I TCP transcription factors, a subfamily out of which several members have been reported to participate in auxin homeostasis in the Arabidopsis gynoecium by binding the IAA3 promoter [61, 62]. In addition to these two candidate genes pointing out auxin perception and response as essential processes affected in apomictic ovules, plant nitrate transporters are known to interact with a wide range of biomolecules, including hormones, such as NITRATE TRANSPORTER 1.1 (NRT1.1), which shows affinity for IAA [63]. NRTs belong to the large PRT/NRT1 (peptide transporter/nitrate transporter 1) family of transporters that perform essential function during plant development [64]. Several PRT/NRT1 members are involved in early embryogenesis and gametophyte development in Arabidopsis [65, 66], including the homolog of OsNRT1.1A, whose loss of function provokes embryo arrest [67]. Furthermore, several transporter genes for different biomolecules (amino acid, sugars, ions) targeted by small RNAs also showed significant decrease in expression.

In summary, sRNA, miRNA and target analyses identified 14 molecules related with auxin transport, metabolism and response and encoded by a gene that differed in expression between sexual and apomictic plants, including: AMINO ACID/AUXIN PERMEASE 15 (auxin influx carrier) [68]; INDOLE-3-ACETIC ACID (IAA)AMIDO SYNTHETASE GH3-8 (catalyzes the synthesis of indole-3-acetic acid (IAA)-amino acid conjugates, providing a mechanism for the plant to cope with the presence of excess auxin) [69]; IAA30 (repressor of early auxin response genes at low auxin concentrations) [70]; miR160 and miR167 (regulate expression of ARFs auxin response factors) [69]; miR164 (related with TCPcontrolled auxin response) [49-51]; targets ARF2; ARF8; ARF10; ARF12 (auxin response factors) [54, 55]; AFB2 (AUXIN SIGNALING F-Box 2) [71]; PROLIFERATING CELL FACTOR 6 (TCP family) (auxin homeostasis, IAA3 promoter binding) [61, 62]; NITRATE TRANSPORTER 1.1 (NRT1.1) [63]. Moreover, a comparative analysis of the apomictic and sexual Paspalum floral reference transcriptomes revealed more 40 genes related to auxin metabolism and signaling showing differential expression [24]. Altogether, these findings suggests that auxin metabolism and response might be perturbed during reproductive development in $P$. notatum apomictic biotypes, resulting in altered signaling pathways and ultimately affecting growth and development.

\section{Conclusions}

Our analysis of the small RNA expressed on the floral transcriptome of sexual and apomictic genotypes indicated that numerous genes belonging to PO ontology classes associated with reproductive development are probably controlled by epigenetic mechanisms and differentially expressed between both reproductive types. Moreover, the analysis of the small reads data set combining several Gramineae genome references and Paspalum transcriptomic resources allowed the detection of miRNAs from 22 known conserved plant families, predicted novel members for two conserved plant families (miR156 and miR171) and four novel Gramineae 
miRNA families, including one specific to $P$. notatum. Furthermore, an integration of differential representation analyses for small RNAs, miRNA genes and miRNA targets suggested that small RNA-dependent auxincentered mechanisms might be involved in the switch from sexuality to apomixis. The differences we observed in small RNA targets and miRNA biogenesis provide novel, attractive hypotheses that will guide future functional research. With regards to this, addressing the roles of miR160, miR167 and miR319 during sexual reproduction appears critical to expand our understanding of the molecular mechanisms underlying apomixis in Paspalum species.

\section{Methods}

\section{Plant material}

Two Paspalum notatum tetraploid $(2 \mathrm{n}=4 \mathrm{x}=40)$ genotypes were used to collect material for small RNA libraries construction: Q4117, a highly heterozygous natural accession collected from the State of Rio Grande do Sul (Southern Brazil) that reproduces through obligate aposporous apomixis [72] and C4-4x, a fully sexual genotype experimentally obtained from chromosome duplication of a highly heterozygous sexual diploid by colchicine treatment [30]. Due to the heterozygous nature of the diploid of origin, many of the C4-4x loci are expected to be heterozygous as well. However, a lower degree of heterozygosity is expected with respect to Q4117. P. notatum is a perennial rhizomatous species, therefore allowing multiplication through vegetative replicas. Both genotypes (Q4117 and C4-4x) belong to the living Paspalum germplasm collection established at IBONE-CONICET-UNNE (Instituto de Botánica del Nordeste, Corrientes, Argentina). Vegetative replicates were transferred and maintained in experimental plots at IICARCONICET-UNR (Instituto de Investigaciones en Ciencias Agrarias de Rosario, Zavalla, Argentina). Replicates of both Q4117 and C4-4x were established in neighboring plots in order to guarantee identical growing environmental conditions.

\section{Library preparation, sequencing and quality control}

Small RNAs libraries were produced in triplicate from evenly balanced bulks (i. e. balanced mixes) of spikelets collected at four developmental stages: premeiosis, meiosis, postmeiosis and anthesis. The material was classified into different developmental stages categories by correlating micro- and megagametophyte development and following the $P$. notatum reproductive calendar, as recommended in previous work [20]. Equivalent amounts (mg) of plant material (spikelets) from each developmental stage were mixed in order to produce two bulks (apomictic and sexual), whose comparison is expected to reveal contrasts between reproductive modes, yet it will not provide temporal resolution. Briefly, total RNA was extracted using the SV Total RNA
Isolation System (Promega). RNA samples were then quantified by fluorescence (Qubit 2.0 Fluorometer, Thermo Fisher Scientific) and RNA integrity was determined using an Agilent Bioanalyzer 2100. Small RNA libraries were prepared following the Illumina TruSeq Small RNA sample preparation guide and 50-bp single-end sequencing was performed using the Illumina MiSeq system by Polo d'Innovazione di Genomica, Genetica e Biologia (Polo GGB), Siena, Italy. Raw reads were trimmed for adapters and low sequence quality using Cutadapt v1.3 [73], FastQC v.0.11.5 [74] and multiQC [75]. Sequences were finally filtered for 18-26 nt length and for Phred quality score $>25$. The sRNA datasets were deposited in the NCBI Sequence Read Archive (SRA) repository under accession number SRP099144.

\section{Paspalum transcripts annotation and AGO proteins phylogenetic analysis}

We re-annotated the Paspalum transcriptome reference by comparing transcripts with the Oryza sativa Japonica reference peptides (genome assembly IRGPS-1.0) provided by the Gramene database [76]. For global annotation, we retained Blastx best alignments with an e-value $<0.05$ and an identity of at least 50\%, resulting in 33,071 annotated isogroups/genes (out of 51,168). More stringent criteria were used for searching genes with annotations related to small RNA-directed pathways (e-value $<1 \mathrm{e}^{-05}$, ID $>50 \%$, and alignment length $>200$ ). For AGO proteins phylogenetic analysis, we used the MUSCLE alignment and the Neighbor-joining options of Geneious ${ }^{\circ} 10.0 .9$. Phylogenetic tree was inferred after careful visual inspection and postprocessing multiple alignments for masking gaps and using bootstrap values (1000 replicates). A. thaliana and O. sativa Japonica sequences were recovered from the Gramene database [76] and $P$. notatum protein sequences were predicted from selected transcripts.

\section{Small RNA reads alignments and representation over the $P$. notatum transcriptome}

Small RNA reads were aligned onto the 454/Roche $\mathrm{FLX}+P$. notatum reference floral transcriptome generated from Q4117 and C4-4x genotypes (referred to as Paspalum reference transcriptome) [24]. Mapping was performed using the Bowtie aligner [77] with the following parameters: no mismatch allowed $(-n 0), 19$ high quality bases required to initiate the alignment $(-119)$ and reporting the best alignments only ('best' option). Read counting for each reference transcript was performed with the feature counts module of Subread [78] using $\mathrm{Q}=10$ and no strand selection. Normalization of reads counts over transcripts was carried out using the upper-quartile method option of edgeR [79]. Only transcripts showing a normalized count value per millions (cpm) of 1 , in at least two samples were considered for further analyses. The edgeR package [79] was used to 
analyze differential expression following a glm approach [79]. Sexual samples were established as controls, therefore positive values of $\operatorname{logFC}$ were associated to an increased number of sRNA reads in apomictic libraries and negative values indicated an increased number of sRNA reads in sexual libraries. Read coverage comparison resulting in an adjusted $p$-value $<0.05$ (FDR) [80]. Comparisons producing FDR $<0.05$ and fold changes in the range of 1-2 and $>2$ were considered as moderate and high coverage differences, respectively.

\section{Identification of conserved and novel microRNA loci expressed in the floral transcriptome of $P$. notatum} In order to predict miRNA genes expressed in the P. notatum floral transcriptome, small RNA sequences were analyzed with ShortStack version v05 using default parameters [81]. Since no genomic reference is available for Paspalum, small RNA alignments were performed using the Paspalum reference transcriptome and three other genomic sequences of related grasses including: Oryza sativa Japonica (Assembly GCA_001433935.1), Sorghum bicolor v2 (Assembly: GCA_000003195.2) and Setaria italica JGIv2.0 (Assembly GCA_000263155.2). Briefly, we aligned the small RNA reads against each reference using Bowtie allowing two mismatches (-n 2) and requiring 18 high quality bases to initiate the alignment $(-1$ 18). Then, candidate cluster (i.e. genome/transcriptome-wide regions containing small RNAs accumulation) associated to small RNA sequences were identified with the default parameters. Putative miRNAs were then selected based on a DicerCall of 20-22. Mature miRNA sequences were compared against miRBase [48] using the SSEARCH option and a cut off E-value $<0.05$ to detect conserved miRNAs. Mature sequences that produced no hit were considered as putative new $P$. notatum miRNAs (Pn_miRNAs). The secondary structure of the predicted novel miRNA genes was analyzed using RNAfold within the ViennaRNA Package [82] and manually examined for the presence of putative mature miRNA sequences in one arm of the stem region, bulges with no more than 3 unpaired nucleotides, a loop of at least 10 nucleotides in length and free-folding energy lower than or equal to $-60 \mathrm{kcal} \mathrm{mol}^{-1}$ [83]. Multiple mature sequence alignments were performed with the software Mega6 [84]. The phylogenetic relationships among sequences were reconstructed using the UPGMA method with default parameters and assessed using 1000 bootstrap replications.

\section{Expression analyses of miRNA precursors}

Differential expression of miRNA precursors was carried out using edgeR [79]. Only 20-24 nt reads with counts $\geq 5$ were retained for the analysis. Read counts over precursors were normalized by library size, using the default TMM (trimmed mean of M-values) method [79]. Normalized read numbers on each precursor in apomictic and sexual samples were compared using the sexual sample as a reference (apo vs sex). Thus, positive and negative $\log \mathrm{FC}$ values indicated higher and lower miRNAs expression levels, respectively. Sequences with corrected $p$ values $(\mathrm{FDR}) \leq 0.05$ and $\log \mathrm{FC} \geq|1|$ were considered differentially expressed.

\section{Identification of miRNA target transcripts in Paspalum and differential expression}

We used TargetFinder [85] with the default parameters and a cut off value of 4 to predict miRNAs targets expressed in the Paspalum reference transcriptome. Common ontologies (GO-Slim and PO) were established using the PANTHER [86] and Planteome [45]. Finally, for differential expression of Paspalum targets between sexual and apomictic developments, we took advantage of former expression analysis, considering only those transcripts that showed at least 10 reads in one of the libraries [24].

\section{Additional files}

Additional file 1: Lists of annotated Paspalum floral transcripts involved in sRNA-directed pathways. Section 1. List of key rice genes involved in small RNA-directed pathways and their P. notatum homologs expressed in floral tissues. Section 2: Expression levels of floral $P$. notatum transcripts homologous to key genes involved in small RNA-directed pathways. (XLSX $22 \mathrm{~kb}$ )

Additional file 2: General statistics for the sequencing of triplicate floral sRNA libraries. The sRNA libraries were originated from apomictic (Apo) and sexual (Sex) Paspalum notatum genotypes. (PDF $178 \mathrm{~kb}$ )

Additional file 3: Lists of $P$. notatum floral transcripts harboring sRNA derived from apomictic and sexual libraries. Section 1: Number of small RNA reads from apomictic (Apo) and sexual (Sex) libraries mapping onto the Paspalum transcriptome reference. Section 2: List of the $1000 P$. notatum transcripts with highest small RNA coverage. Section 3: Classification of the Paspalum annotated small RNA targets into ontology classes, according to Plant Structure Development Stage Ontologies (www.planteome.org). Section 4. Functional classification and PANTHER overrepresentation test for annotated small RNA targets. Section 5: edgeR analysis of small RNA reads mapped over the $P$. notatum reference transcriptome. (XLSX $6165 \mathrm{~kb}$ )

Additional file 4: Lists of miRNA detected in floral samples of sexual and apomictic $P$. notatum genotypes. Section 1. List of clusters harboring miRNAs detected in four references ( $P$. notatum floral transcriptome, O. sativa genome, S. italica genome, S. bicolor genome) with ShortStack v.3.8.5. Section 2. BLAST SSEARCH of mature miRNAs sequences of $P$. notatum against MirBase. Section 3. edgeR expression analysis of clusters harbouring miRNAs detected using the abovementioned four references. (XLSX $51 \mathrm{~kb}$ )

Additional file 5: Folding analysis of miRNA putative precursors. Secondary structures derived from folding 8 predicted miRNA precursors. (PDF 667 kb)

Additional file 6: Phylogenetic analysis of miRNA sequences. Phylogenetic analysis by maximum likelihood method of mature miRNA sequences expressed in the $P$. notatum floral transcriptome. (PDF 53 kb)

Additional file 7: miRNA targets prediction. Section 1: miRNA targets detected in the Paspalum transcriptome reference and annotated after blastx on a rice peptide database ( $E-v a l<0.05$ and $\%$ identity $>50$ ). Section 2: miRNA targets with or without differential expression between sexual and apomictic libraries, as detected in the Paspalum transcriptome reference: read coverage analysis and annotation. (XLSX 68 kb) 


\section{Abbreviations}

ARF: Auxin response factor; dsRNA: Double-stranded RNA; ES: Embryo sac; FC: Fold change; FDR: False discovery rate; INDEL: Insertion/deletion; miRNA: Micro RNA; mRNA: Messenger RNA; PTGS: Post-transcriptional gene silencing; RdDM: RNA-dependent DNA methylation; RDR: RNA-dependent RNA polymerase; SNP: Single nucleotide polymorphism; sRNA: Small RNA

\section{Acknowledgments}

Authors acknowledge to Professor Camilo Quarin from IBONE for providing the plant material used in this work. C. Azzaro and C. Colono received fellowships from CONICET, Argentina. J.P.A. Ortiz, L. Siena, M. Podio and S. Pessino are research staff members of CONICET, Argentina.

\section{Authors' contributions}

JPAO designed the experiments, performed the bioinformatics analysis and wrote the manuscript. OL performed the phylogenetic and bioinformatics analysis, the interpretation of results, and the manuscript writing. CR and MG did the sequencing experiments and participated in the bioinformatics analysis. LAS carried out flower collection and classification, extracted the RNA and contributed to prepare the libraries. MP, CC, CA were involved in material collection and classification. SCP designed the experiments, carried out flower collection and classification, RNA extraction, interpretation of results and manuscript writing. All authors read and approved the final manuscript.

\section{Funding}

This work was funded by the European Union's Horizon 2020 Research and Innovation Program under the Marie Skłodowska-Curie Grant Agreement No 645674; Agencia Nacional de Promoción Científica y Tecnológica (ANPCyT), Argentina, Project PICT-2014-1080; Consejo Nacional de Investigaciones Científicas y Técnicas (CONICET), Argentina, Project PIP 11220090100613; Universidad Nacional de Rosario (UNR), Argentina, Project: AGR271.

\section{Availability of data and materials}

The datasets supporting the conclusions of this article are available in the NCBI Sequence Read Archive (SRA) repository under accession number SRP099144. Additional dataset(s) supporting the conclusions of this article are included within the article and its additional files. The plant materials used in this study belong to the living germplasm collection of Instituto de Botánica del Nordeste (IBONE), CONICET-UNNE, Corrientes, Argentina. Voucher specimens of this material are deposited at the Herbarium CTES-IBONE (publicly available), under deposition numbers: C4-4X (Quarin, C. L. 4260, barcode CTES0541627, cardboard No. 330064); Q4117 (Quarin, C. L. 4117, barcode CTES0541626, cardboard No. 233851).

\section{Ethics approval and consent to participate}

Not applicable.

\section{Consent for publication}

Not applicable.

\section{Competing interests}

The authors declare that they have no competing interests.

\section{Author details}

${ }^{1}$ Instituto de Investigaciones en Ciencias Agrarias de Rosario (IICAR, CONICET-UNR), Facultad de Ciencias Agrarias, Universidad Nacional de Rosario, Zavalla, Argentina. ${ }^{2}$ Institut de Recherche pour le Développement (IRD), Université de Montpellier, Montpellier, France. ${ }^{3}$ Instituto de Agrobiotecnología de Rosario (INDEAR), Rosario, Argentina.

Received: 5 February 2019 Accepted: 4 June 2019

Published online: 13 June 2019

\section{References}

1. Borges F, Martienssen RA. The expanding world of small RNAs in plants. Nat Rev Mol Cell Biol. 2015;16:727.

2. Zhang H, Lang Z, Zhu JK. Dynamics and function of DNA methylation in plants. Nature Rev Mol Cell Biol. 2018;19:489-506.

3. Martínez G, Köhler C. Role of small RNAs in epigenetic reprogramming during plant sexual reproduction. Curr Opin Plant Biol. 2017;36:22-8.
4. Nonomura KI. Small RNA pathways responsible for non-cell autonomous regulation of plant reproduction. Plant Reprod. 2018;31:21-9.

5. Armenta-Medina A, Demesa-Arévalo E, Vielle-Calzada JP. Epigenetic control of cell specification during female reproduction. Sex Plant Reprod. 2011;24: 137-47.

6. Nogler G. Gametophytic apomixis. In: Johri BM, editor. Embryology of angiosperms. Berlin: Springer; 1984. p. 475-518.

7. Grimanelli D, Leblanc O, Perotti E, Grossniklaus U. Developmental genetics of gametophytic apomixis. Trends Genet. 2001;17:597-604.

8. Asker S, Jerling L. Apomixis in plants. Boca Raton and London: CRC press; 1992.

9. Spillane C, Curtis MD, Grossniklaus U. Apomixis technology developmentvirgin births in farmers' fields? Nat Biotechnol. 2004;22:687-91.

10. Spielman M, Vinkenoog R, Scott RJ. Genetic mechanisms of apomixis. Philos T Roy Soc B. 2003;358:1095-103.

11. Leblanc O, Grimanelli D, Hernandez-Rodríguez M, Galindo PA, SorianoMartínez AM, Perotti E. Seed development and inheritance studies in apomictic maize-Tripsacum hybrids reveal barriers for the transfer of apomixis into sexual crops. Int J Dev Biol. 2009;53:585-96.

12. Hand ML, Koltunow AM. The genetic control of apomixis: asexual seed formation. Genetics. 2014;197:441-50.

13. Ozias-Akins P. Apomixis: developmental characteristics and genetics. Crit Rev Plant Sci. 2006;25:199-214.

14. Barcaccia G, Albertini E. Apomixis in plant reproduction: a novel perspective on an old dilemma. Plant Reprod. 2013;26:159-79.

15. Ozias-Akins P, Van Dijk PJ. Mendelian genetics of apomixis in plants. Annu Rev Genet. 2007;41:509-37.

16. Conner JA, Mookkan M, Huo H, Chae K, Ozias-Akins P. A parthenogenesis gene of apomictic origin elicits embryo formation from unfertilized eggs in a sexual plant. Proc Nat Acad Sci. 2015;112:11205-10.

17. Siena LA, Ortiz JPA, Calderini O, Paolocci F, Caceres ME, Kaushal P, et al. An apomixis-linked ORC3-like pseudogene is associated with silencing of its functional homolog in apomictic Paspalum simplex. J Exp Bot. 2016;67:1965-78.

18. Mancini M, Permingeat H, Colono C, Siena L, Pupilli F, Azzaro C, et al. The MAP3K-coding QUI-GON JINN (QGJ) gene is essential to the formation of unreduced embryo sacs in Paspalum. Front Plant Sci. 2018. https://doi.org/ 10.3389/fpls.2018.01547.

19. Albertini E, Marconi G, Barcaccia G, Raggi L, Falcinelli M. Isolation of candidate genes for apomixis in Poa pratensis L. Plant Mol Biol. 2004;56:879-94.

20. Laspina NV, Vega T, Seijo G, González AM, Martelotto LG, Stein J, et al. Gene expression analysis at the onset of aposporous apomixis in Paspalum notatum. Plant Mol Biol. 2008;67:615-28.

21. Sharbel TF, Voigt ML, Corral JM, Galla G, Kumlehn J, Klukas C, et al. Apomictic and sexual ovules of Boechera display heterochronic global gene expression patterns. Plant Cell. 2010;22:655-71.

22. Silveira ED, Guimarães LA, Dusi DMA, Da Silva FR, Martins NF, Do Carmo Costa MM, et al. Expressed sequence-tag analysis of ovaries of Brachiaria brizantha reveals genes associated with the early steps of embryo sac differentiation of apomictic plants. Plant Cell Rep. 2012;31:403-16.

23. Okada T, Hu Y, Tucker MR, Taylor JM, Johnson SD, Spriggs A, et al. Enlarging cells initiating apomixis in Hieracium praealtum transition to an embryo sac program prior to entering mitosis. Plant Physiol. 2013;163:216-31.

24. Ortiz JPA, Revale S, Siena LA, Podio M, Delgado L, Stein J, et al. A reference floral transcriptome of sexual and apomictic Paspalum notatum. BMC Genomics. 2017;18:318.

25. Koltunow AM, Grossniklaus U. Apomixis: a developmental perspective. Ann Rev Plant Biol. 2003;54:547-74.

26. Pupilli F, Barcaccia G. Cloning plants by seeds: inheritance models and candidate genes to increase fundamental knowledge for engineering apomixis in sexual crops. J Biotech. 2012;159:291-311.

27. Podio M, Cáceres ME, Samoluk SS, Seijo JG, Pessino SC, Ortiz JPA, et al. A methylation status analysis of the apomixis-specific region in Paspalum spp. suggests an epigenetic control of parthenogenesis. J Exp Bot. 2014;65:6411-24.

28. Zappacosta DC, Ochogavía AC, Rodrigo JM, Romero JR, Meier MS, Garbus I, et al. Increased apomixis expression concurrent with genetic and epigenetic variation in a newly synthesized Eragrostis curvula polyploid. Sci Rep. 2014:4:4423.

29. Rodrigo JM, Zappacosta DC, Selva JP, Garbus I, Albertini E, Echenique V. Apomixis frequency under stress conditions in weeping lovegrass (Eragrostis curvula). PLoS One. 2017;12:e0175852. 
30. Quarin CL, Espinoza F, Martínez EJ, Pessino SC, Bovo OA. A rise of ploidy level induces the expression of apomixis in Paspalum notatum. Sex Plant Reprod. 2001;13:243-9.

31. Ochogavía AC, Cervigni G, Selva JP, Echenique VC, Pessino SC. Variation in cytosine methylation patterns during ploidy level conversions in Eragrostis curvula. Plant Mol Biol. 2009;70:17-29.

32. Rodríguez MP, Cervigni GDL, Quarin CL, Ortiz JPA. Frequencies and variation in cytosine methylation patterns in diploid and tetraploid cytotypes of Paspalum notatum. Biol Plantarum. 2012;56:276-82.

33. García-Aguilar M, Michaud C, Leblanc O, Grimanelli D. Inactivation of a DNA methylation pathway in maize reproductive organs results in apomixis-like phenotypes. Plant Cell. 2010;22:3249-67.

34. Olmedo-Monfil V, Durán-Figueroa N, Arteaga-Vázquez $M$, Demesa-Arévalo $E$, Autran D, Grimanelli D, et al. Control of female gamete formation by a small RNA pathway in Arabidopsis. Nature. 2010;464:628.

35. Singh M, Goel S, Meeley RB, Dantec C, Parrinello H, Michaud C, et al. Production of viable gametes without meiosis in maize deficient for an ARGONAUTE protein Plant Cell. 2011;23:443-58.

36. Hernández-Lagana E, Rodríguez-Leal D, Lúa J, Vielle-Calzada JP. A multigenic network of ARGONAUTE4 clade members control early megaspore formation in Arabidopsis. Genetics. 2016;204:1045-56.

37. Selva JP, Siena L, Rodrigo JM, Garbus I, Zappacosta D, Romero JR, et al. Temporal and spatial expression of genes involved in DNA methylation during reproductive development of sexual and apomictic Eragrostis curvula. Sci Rep. 2017;7:15092.

38. Amiteye S, Corral JM, Vogel H, Sharbel TF. Analysis of conserved microRNAs in floral tissues of sexual and apomictic Boechera species. BMC Genomics. 2011;12:500.

39. Galla G, Volpato M, Sharbel T, Barcaccia G. Computational identification of conserved microRNAs and their putative targets in the Hypericum perforatum L. flower transcriptome. Plant Reprod. 2013;26:209-29.

40. Rabiger DS, Taylor JM, Spriggs A, Hand ML, Henderson ST, Johnson SD, et al. Generation of an integrated Hieracium genomic and transcriptomic resource enables exploration of small RNA pathways during apomixis initiation. BMC Biol. 2016;14:86.

41. Long JM, Liu Z, Wu XM, Fang YN, Jia HH, Xie ZZ, et al. Genome-scale mRNA and small RNA transcriptomic insights into initiation of citrus apomixis. J Exp Bot. 2016;67:5743-56.

42. Ortiz JPA, Quarin CL, Pessino SC, Acuña C, Martínez EJ, Espinoza F, et al. Harnessing apomictic reproduction in grasses: what we have learned from Paspalum. Ann Bot. 2013;112:767-87.

43. Fang X, Qi Y. RNAi in plants: an ARGONAUTE-centered view. Plant Cell. 2016;28:272-85.

44. Ma L, Hatlen A, Kelly $\sqcup$, Becher $H$, Wang W, Kovarik A, et al. Angiosperms are unique among land plant lineages in the occurrence of key genes in the RNA-directed DNA methylation (RdDM) pathway. Genome Biol Evol. 2015;7:2648-62

45. Cooper L, Meier A, Laporte MA, Elser JL, Mungall C, Sinn BT, et al. The Planteome database: an integrated resource for reference ontologies, plant genomics and phenomics. Nuc Acids Res. 2018;46:1168-80.

46. Pupilli F, Martínez EJ, Busti A, Calderini O, Quarin CL, Arcioni S. Comparative mapping reveals partial conservation of synteny at the apomixis locus in Paspalum spp. Mol Genet Genomics. 2004;270:539-48.

47. Podio M, Rodríguez MP, Felitti S, Stein J, Martínez EJ, Siena LA, et al. Sequence characterization, in silico mapping and cytosine methylation analysis of markers linked to apospory in Paspalum notatum. Genet Mol Biol. 2012;35:827-37.

48. Griffiths-Jones S. miRBase: the microRNA sequence database. Methods Mol Biol. 2006;342:129-38.

49. Zhai J, Zhang H, Arikit S, Huang K, Nan GL, Walbot V, et al. Spatiotemporally dynamic, cell-type-dependent premeiotic and meiotic phasiRNAs in maize anthers. Proc Natl Acad Sci U S A. 2015;112:3146-51.

50. Fei $Q$, Yang $L$, Liang $W$, Zhang D, Meyers BC. Dynamic changes of small RNAs in rice spikelet development reveal specialized reproductive phasiRNA pathways. J Exp Bot. 2016;67:6037-49.

51. Liu H, Yu H, Tang G, Huang T. Small but powerful: function of microRNAs in plant development. Plant Cell Rep. 2018;37:515-28.

52. Samad AFA, Sajad M, Nazaruddin N, Fauzi IA, Murad AMA, Zainal Z, Ismail I. MicroRNA and transcription factor: key players in plant regulatory network. Front Plant Sci. 2017;85:65

53. Hübner M, Galante PAF, Kreth S, Hinske LC. Identification and validation of potential differential miRNA regulation via alternative polyadenylation. Methods Mol Biol. 2018;1733:87-92.
54. Wang R, Estelle M. Diversity and specificity: auxin perception and signaling through the TIR1/AFB pathway. Curr Opin Plant Biol. 2014;21: $51-88$.

55. Li SB, Xie ZZ, Hu CG, Zhang JZ. A review of auxin response factors (ARFs) in plants. Front Plant Sci. 2016;7:47.

56. Szemenyei $H$, Hannon M, Long JA. TOPLESS mediates auxin-dependent transcriptional repression during Arabidopsis embryogenesis. Science. 2008; 319:1384-6.

57. Pagnussat GC, Alandete-Saez M, Bowman JL, Sundaresan V. Auxindependent patterning and gamete specification in the Arabidopsis female gametophyte. Science. 2009;324:1684-9.

58. Wu Y, Yang L, Yu M, Wang J. Identification and expression analysis of microRNAs during ovule development in rice (Oryza sativa) by deep sequencing. Plant Cell Rep. 2017;36:1815-27.

59. Li S. The Arabidopsis thaliana TCP transcription factors: a broadening horizon beyond development. Plant Signal Behav. 2015;10:e1044192.

60. Tatematsu K, Nakabayashi K, Kamiya Y, Nambara E. Transcription factor AtTCP14 regulates embryonic growth potential during seed germination in Arabidopsis thaliana. Plant J. 2008;53:42-52.

61. Uberti-Manassero NG, Lucero LE, Viola IL, Vegetti AC, Gonzalez DH. The class I protein AtTCP15 modulates plant development through a pathway that overlaps with the one affected by CIN-like TCP proteins. J Exp Bot. 2012;63: 809-23.

62. Lucero LE, Uberti-Manassero NG, Arce AL, Colombatti F, Alemano SG, Gonzalez DH. TCP15 modulates cytokinin and auxin responses during gynoecium development in Arabidopsis. Plant J. 2015;8:267-82.

63. Léran S, Varala K, Boyer JC, Chiurazzi M, Crawford N, Daniel-Vedele F, et al. A unified nomenclature of NITRATE TRANSPORTER 1/PEPTIDE TRANSPORTER family members in plants. Trends Plant Sci. 2014;19:5-9.

64. Tegeder M. Transporters for amino acids in plant cells: some functions and many unknowns. Curr Opin Plant Biol. 2012;15:315-21.

65. Léran S, Garg B, Boursiac Y, Corratgé-Faillie C, Brachet C, Tillard P, et al. AtNPF5.5, a nitrate transporter affecting nitrogen accumulation in Arabidopsis embryo. Sci Rep. 2015;5:7962.

66. Stacey MG, Osawa H, Patel A, Gassmann W, Stacey G. Expression analyses of Arabidopsis oligopeptide transporters during seed germination, vegetative growth and reproduction. Planta. 2006;223:291-305.

67. Almagro A, Lin SH, Tsay YF. Characterization of the Arabidopsis nitrate transporter NRT1.6 reveals a role of nitrate in early embryo development. Plant Cell. 2008;20:3289-99.

68. Young GB, Jack DL, Smith DW, Saier MH. The amino acid/auxin: symport permease family. Biochimica et Biophysica Acta (BBA)- Biomembranes. 1999; 1415:306-22.

69. Staswick PE, Serban B, Rowe M, Tiryaki I, Maldonado MT, Maldonado MC, Suza W. Characterization of an Arabidopsis enzyme family that conjugates aminoacids to indole-3-acetic acid. Plant Cell. 2005;17(2):616-27.

70. Liscum E, Reed JW. Genetics of aux/IAA and ARF action in plant growth and development. Plant Mol Biol. 2002;49:387-400.

71. Dharmasiri N, Dharmasiri S, Weijers D, Lechner E, Yamada M, Hobbie L, Ehrismann JS, Juergens G, Estelle M. Plant development is regulated by a family of auxin receptor F box proteins. Dev Cell. 2005;9:109-19.

72. Ortiz JPA, Pessino SC, Leblanc O, Hayward MD, Quarin CL. Genetic fingerprinting for determining the mode of reproduction in Paspalum notatum, a subtropical apomictic forage grass. Theor Appl Genet. 1997;95: 850-6.

73. Marcel M. Cutadapt removes adapter sequences from high-throughput sequencing reads. EMBnet J. 2011;17:10-2.

74. Andrews S. FastQC: a quality control tool for high throughput sequence data. Available online at: http://www.bioinformatics.babraham.ac.uk/ projects/fastqc. 2010.

75. Ewels P, Magnusson M, Lundin S, Käller M. MultiQC: summarize analysis results for multiple tools and samples in a single report. Bioinformatics. 2016;32:3047-8.

76. Tello-Ruiz MK, Naithani S, Stein JC, Gupta P, Campbell M, Olson A, et al. Gramene 2018: unifying comparative genomics and pathway resources for plant research. Nuc Acids Res. 2018:4:1181-9.

77. Langmead B, Trapnell C, Pop M, Salzberg SL. Ultrafast and memory-efficient alignment of short DNA sequences to the human genome. Genome Biol. 2009;10:R25.

78. Liao Y, Smyth GK, Shi W. The subread aligner: fast, accurate and scalable read mapping by seed-and-vote. Nuc Acids Res. 2013;41:e108. 
79. Robinson MD, McCarthy DJ, Smyth GK. edgeR: a Bioconductor package for differential expression analysis of digital gene expression data. Bioinformatics. 2010;26:139-40.

80. Benjamini $Y$, Hochberg $Y$. On the adaptive control of the false discovery fate in multiple testing with independent statistics. J Educ Behav Stat. 2000;25: 60-83.

81. Axtell MJ. ShortStack: comprehensive annotation and quantification of small RNA genes. RNA. 2013;19:740-51.

82. Lorenz R, Bernhart SH, Höner zu Siederdissen C, Tafer H, Flamm C, Stadler PF, et al. ViennaRNA Package 2.0. Algorithms Mol Biol. 2011;6:1-26.

83. Ambros V, Bartel B, Bartel DP, Burge CB, Carrington JC, Chen X, et al. A uniform system for microRNA annotation. RNA. 2014;9:277-9.

84. Tamura K, Stecher G, Peterson D, Filipski A, Kumar S. MEGA6: molecular evolutionary genetics analysis version 6.0. Mol Biol Evol. 2013;30:2725-9.

85. Fahlgren N, Carrington JC. miRNA target prediction in plants. Methods $\mathrm{Mol}$ Biol. 2010;592:51-7.

86. Mi H, Muruganujan A, Casagrande JT, Thomas PD. Large-scale gene function analysis with the PANTHER classification system. Nat Protoc. 2013;8: 1551-66.

\section{Publisher's Note}

Springer Nature remains neutral with regard to jurisdictional claims in published maps and institutional affiliations.

Ready to submit your research? Choose BMC and benefit from:

- fast, convenient online submission

- thorough peer review by experienced researchers in your field

- rapid publication on acceptance

- support for research data, including large and complex data types

- gold Open Access which fosters wider collaboration and increased citations

- maximum visibility for your research: over $100 \mathrm{M}$ website views per year

At BMC, research is always in progress.

Learn more biomedcentral.com/submissions 